\title{
2 Lernmanagementsysteme als Rahmenbedingung
}

\author{
Matthias Kunkel
}

Im optes-Projekt wurden eine Vielzahl von inhaltlichen Angeboten entwickelt, die zur Durchführung unterschiedliche softwaretechnische Unterstützung brauchen. Aufgebaut wurde zum Beispiel ein Mathematik-Propädeutikum aus thematisch abgestimmten Vorkursen, in denen Lernmodule und Glossare zur Wissensvermittlung und Tests zum Üben und zum Überprüfen des individuellen Lernstands verwendet werden (siehe Kapitel 14). Elektronische Portfolios wurden benötigt, um den Reflexionsprozess der Lernenden zu unterstützen und ihre Kenntnisse und Fähigkeiten im Rahmen des E-Tutoring-Ansatzes zu dokumentieren (siehe Kapitel 5). Für E-Klausuren (siehe Kapitel 11) brauchte es ebenso ein mächtiges Test-Tool wie für den Freien Trainingsplatz (siehe Kapitel 4), auf dem Anwender*innen ihre Fähigkeit zum Lösen mathematischer Aufgaben trainieren können. Dies sind nur einige Beispiele.

Für jedes der genannten Angebote hätte das Projektteam ein eigenständiges, dafür spezialisiertes Software-Tool finden und einsetzen können. Allerdings sah der in optes verfolgte Ansatz auch vor, dass diese Angebote miteinander verknüpft sind. Der Test sollte zum Beispiel Daten auf Basis gegebener Antworten an das Kompetenzmanagement von ILIAS übermitteln, aus dem das Portfolio wiederum eine Visualisierung des Kompetenzstands jeder anwendenden Person generieren kann. Oder eine ursprünglich für den Vorkurs erstellte Frage sollte auch in einer E-Klausur oder im Freien Trainingsplatz wiederverwendet werden können. Damit existierten zahlreiche Anforderungen an Schnittstellen, an die Wiederverwendbarkeit von Inhalten und Daten und an die Flexibilität der gewählten Softwarelösung. ${ }^{1}$

Mit ILIAS stand schon vor Projektbeginn ein Lernmanagementsystem (LMS) zur freien Verfügung, das diese verschiedenen Prozesse bereits unterstützte oder für das mit überschaubarem Aufwand die noch nicht vorhandene Funktionalität umsetzbar war. An Stelle einer Vielzahl unterschiedlicher Softwareanwendungen mit unterschiedlicher Ergonomie, eigener Benutzerverwaltung und divergierender

1 Die starke Verzahnung der verschiedenen Angebote und der dafür genutzten Funktionen ist auch der Grund, warum in optes keine SCORM-Lernmodule verwendet werden - obwohl das dem Wunsch der Wiederverwendung von optes-Angeboten entgegenkäme. Aber im Gegensatz zu den verwendeten ILIAS-Lernmodulen unterstützen SCORM-Module vom Standard her genau nicht die gewünschte Vernetzung von Objekten. Ziel ist es vielmehr, die Abhängigkeit eines Lernmoduls von anderen Anwendungen möglichst gering zu halten und dessen Wiederverwendung in verschiedensten Kontexten zu ermöglichen. 
Softwareanforderungen, konnte das optes-Gesamtvorhaben mit Hilfe von ILIAS auf einer einzigen softwaretechnischen Plattform entwickelt und bereitgestellt werden.

Die Vorteile dieser Lösung waren nicht nur eine einheitliche Benutzerführung in allen Teilbereichen der Plattform, eine gemeinsame Benutzerverwaltung und Zugriffskontrolle für alle Anwendungen sowie ein überschaubarer Aufwand für die Systemadministration, da nur eine Softwareanwendung betreut werden musste. Dass es für die Nutzung der optes-Angebote nur eines Lernmanagementsystems bedarf, war auch für die Weitergabe der optes-Angebote im Zuge der Dissemination der Projektergebnisse von entscheidendem Vorteil.

Anwender*innen, deren Hochschule bereits ILIAS nutzten, konnten relativ einfach die optes-Angebote in ihre bestehende Plattform integrieren und verwenden. ${ }^{2}$ Anwender*innen, die noch kein LMS besaßen, mussten zur Nutzung von optes nur ILIAS installieren und die optes-Angebote importieren. Und Anwender*innen, die ein anderes LMS verwendeten, konnten eine ILIAS-Installation parallel zu ihrer zentralen Lernplattform betreiben und die optes-Angebote per LTISchnittstelle ${ }^{3}$ in ihr LMS einbinden.

Durch die Verwendung eines Standard-Lernmanagementsystems, dessen Entwicklung unabhängig vom optes-Projekt verläuft, wurde außerdem die Nachhaltigkeit der Nutzung der optes-Angebote gesichert. Denn auch nach dem Ende der Projektförderung von optes wird ILIAS durch seine breit aufgestellte Community weiterentwickelt und genutzt. Die in ILIAS entwickelten Angebote sind damit auch ohne die Förderung von optes nutzbar und wiederverwendbar. Wäre hingegen für optes eine eigene Softwarelösung entwickelt worden, könnte deren Betrieb und Pflege nach Projektende nicht mehr gewährleistet werden. Zudem wären für eine vollständige Neuentwicklung unverhältnismäßig mehr Finanzmittel notwendig gewesen als für die punktuelle Erweiterung eines an sich schon ausgereiften und leistungsfähigen Systems wie ILIAS.

Die Verwendung eines Standard-Lernmanagementsystems an Stelle einer auf das Projekt optimierten Einzellösung verlangte von den Projektbeteiligten aber auch Kompromisse. So mussten Wünsche an die Weiterentwicklung von ILIAS immer in den regulären Anforderungs- und Abstimmungsprozess des Open-

2 Damit dieser Vorteil zum Tragen kommt, mussten die Angebote in ILIAS für eine Standard-Version des Lernmanagementsystem entwickelt werden und durften kein spezifisch angepasstes ILIAS benötigen. Siehe hierzu auch die Aspekte von Anpassungs- und Kernentwicklung in Kapitel 27.

3 Die Möglichkeit, ILIAS-Kurse und Lerninhalte über eine LTI-Schnittstelle in andere LMS zu provisionieren, steht erst seit Anfang 2018 zur Verfügung und wurde mit der ILIAS-Version 5.3 eingeführt. Hochschulen, die z. B. das an vielen Einrichtungen genutzte LMS Moodle verwenden, können mathematische Vorkurse von optes über die LTI-Schnittstelle in Moodle bereitstellen. Die Studierenden nutzen den Vorkurs dann scheinbar im ihnen bekannten LMS, bewegen sich de facto aber auf dem ILIAS-Server und nutzen die dort vorhandenen Inhalte und Funktionen. 
Source-Projekts eingebracht und verhandelt werden. ${ }^{4}$ Nicht immer konnten sich hierbei die Projektbeteiligten durchsetzen. Oftmals waren auch Zugeständnisse bei Funktionalität und Umsetzung notwendig, damit die neu geschaffene Funktionalität in das Standard-ILIAS passte, nicht eine unnötig hohe Komplexität bei der Bedienbarkeit schuf und die betroffenen Softwarekomponenten auch in anderen Szenarien noch gut funktionierten und nutzbar waren.

Mit der Nutzung einer Standard-Software wie ILIAS an Stelle einer projekteigenen Einzellösung waren alle Teilprojekte auch gezwungen, die Entwicklung ihrer inhaltlichen Angebote an den Release-Zyklus von ILIAS anzupassen. Wurde für optes ein neues Feature in ILIAS entwickelt, stand dieses auf der optes-Plattform wie auf allen anderen ILIAS-Installationen erst dann zur Verfügung, nachdem die neue ILIAS-Version fertig entwickelt, getestet und veröffentlicht wurde. Teilprojekte mussten so mitunter ein Jahr und mehr auf ein benötigtes Feature warten. ${ }^{5}$ Ein entsprechend vorausschauendes Projektmanagement war deshalb nötig.

Dafür profitierte das optes-Projekt im gesamten Förderzeitraum von der dynamischen Entwicklung der Standard-Version von ILIAS. So standen dem Projektteam im Laufe der Zeit verschiedene neue Funktionen zur Verfügung, deren Entwicklung von anderen Anwender*innen finanziert und umgesetzt wurde. Sobald diese Features in den neuen ILIAS-Versionen der Allgemeinheit zur Verfügung standen, waren sie auch für die Umsetzung der optes-Szenarien nutzbar. Hätte es solche Synergieeffekte nicht gegeben, wären für die softwaretechnische Umsetzung der verschiedenen Anwendungsszenarien von optes wesentlich mehr Finanzmittel notwendig gewesen.

Nicht alle gewünschten und von optes benötigten Funktionen konnten aber für den Kern von ILIAS implementiert werden. Zwei für optes und die Nutzung mathematischer Inhalte wesentliche Features wurden als Plug-ins konzipiert und umgesetzt: die Einbindung des STACK-Fragetyps ${ }^{6}$ und die Darstellung geometrischer Objekte mit GeoGebra.

In beiden Fällen wird eine externe und unabhängig von ILIAS existierende Software über eine Plug-in-Schnittstelle in ILIAS eingebunden und nutzbar gemacht. Dieser technische Umstand ist aber weder für die Autor*innen, die Inhalte erstellen, noch für die Lerner*innen, die diese nutzen, spürbar. Wie bei einem vom ILIAS-Kern bereitgestellten Feature wird auch hier wird die Funktionalität der jeweiligen Drittsoftware innerhalb des Lernmanagementsystems bereitgestellt und für die Anwender*innen nutzbar gemacht.

\footnotetext{
${ }^{4}$ Der Softwareentwicklungsprozess von ILIAS und seine Anforderungen und Abläufe wird beschrieben in Kapitel 27.

${ }^{5}$ Kapitel 27 gibt einen Einblick in den Releasezyklus von ILIAS, die zu Projektbeginn genutzte Möglichkeit der beschleunigten Softwareentwicklung mit Release-Branches und den Gründen zum Wechsel auf Standard-Versionen für ILIAS.

${ }^{6}$ Zur Bedeutung von STACK und seiner Nutzung in ILIAS siehe Kapitel 10.
} 
Und auch bei der Weiterentwicklung der STACK-Unterstützung in ILIAS entstanden ähnliche Synergieeffekte wie bei der Entwicklung des Standard-ILIAS. Während die Erstimplementierung von STACK in ILIAS zunächst vor allem von Hochschulen betrieben wurde, die direkt an optes beteiligt waren, übernahmen mit der Zeit andere Hochschulen die Finanzierung, um die Unterstützung von STACK in ILIAS weiter zu verbessern.

Auch wenn ILIAS strengere Rahmenbedingungen für das optes-Projekt geschaffen hat, als dies vielleicht bei einer softwaretechnischen Einzellösung der Fall gewesen wäre - vor allem auf lange Sicht überwiegen die Vorteile durch den Einsatz einer sich kontinuierlich weiterentwickelnden Standardlösung. Und nur mit diesem Ansatz ist eine Weitergabe und Weiternutzung der in optes erstellten Inhalte möglich und damit deren Nachhaltigkeit gesichert.

Open Access Dieses Kapitel wird unter der Creative Commons Namensnennung 4.0 International Lizenz (http://creativecommons.org/licenses/by/4.0/deed. de) veröffentlicht, welche die Nutzung, Vervielfältigung, Bearbeitung, Verbreitung und Wiedergabe in jeglichem Medium und Format erlaubt, sofern Sie den/ die ursprünglichen Autor(en) und die Quelle ordnungsgemäß nennen, einen Link zur Creative Commons Lizenz beifügen und angeben, ob Änderungen vorgenommen wurden.

Die in diesem Kapitel enthaltenen Bilder und sonstiges Drittmaterial unterliegen ebenfalls der genannten Creative Commons Lizenz, sofern sich aus der Abbildungslegende nichts anderes ergibt. Sofern das betreffende Material nicht unter der genannten Creative Commons Lizenz steht und die betreffende Handlung nicht nach gesetzlichen Vorschriften erlaubt ist, ist für die oben aufgeführten Weiterverwendungen des Materials die Einwilligung des jeweiligen Rechteinhabers einzuholen.

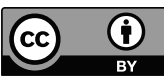

